\title{
Evaluation of Modified Techniques for Toggle Pin Repair of Coxofemoral Luxation in Dogs: A Cadaveric Study
}

\author{
Jacob I. Helmick ${ }^{1}$ Suzanne E. Bugbee ${ }^{1} \quad$ Jason R. Strasberg ${ }^{1} \quad$ Samuel P. Franklin ${ }^{1}$ \\ ${ }^{1}$ The Department of Small Animal Medicine and Surgery, University of \\ Georgia College of Veterinary Medicine, Athens, Georgia, United States \\ Address for correspondence Jacob I. Helmick, DVM, 2200 College \\ Station Road, Athens, GA 30602, United States \\ (e-mail: jacob.helmick25@uga.edu).
}

Vet Comp Orthop Traumatol 2018;31:315-320.

\begin{abstract}
Keywords

- coxofemoral luxation

- hip luxation

- toggle pin

- Arthrex Tightrope

- dogs
\end{abstract}

Objectives The primary objective was to assess acetabular articular cartilage damage when an acetabular tunnel is drilled by passing the drill bit through the femoral tunnel. The second objective was to assess the success rate of passing the toggle through the femoral and acetabular tunnels in a single manoeuvre using a toggle delivery device. Methods Open bilateral coxofemoral luxations in 16 canine cadavers were repaired by toggle pin technique by a surgical resident $(n=8)$ and a diplomate surgeon $(n=8)$. In one hip of each cadaver, the acetabular tunnel was drilled through the femoral tunnel and the toggle was passed in a single manoeuvre using the delivery device. In the contralateral hip, the tunnels were drilled separately, and the toggle was passed through each tunnel separately (resident) or in a single manoeuvre (surgeon). Pelves were examined for acetabular cartilage damage and toggle placement.

Results Cartilage damage did not occur with independent drilling of the acetabulum but occurred in 6/16 hips in which the acetabulum was drilled via the femoral tunnel. Successful deployment of the toggle was obtained in 24/24 hips in which the toggle was passed through the femoral and acetabular tunnels simultaneously using the delivery device, regardless of how the acetabular tunnel was drilled.

Clinical Significance Drilling the acetabular tunnel through the femoral tunnel risks damaging the acetabular cartilage. Success was consistently obtained when passing the toggle through the femoral and acetabular tunnels simultaneously using the toggle delivery device.

\section{Introduction}

Many treatment options for canine coxofemoral luxation have been described including toggle pin fixation. ${ }^{1}$ This technique replaces the round ligament of the femur with a prosthetic ligament; the prosthesis is anchored on the medial side of the acetabular wall and traverses a femoral bone tunnel from the fovea capitis to the proximolateral aspect of the femur. ${ }^{2}$ Although success rates with this surgery are good, failure rates as high as 11 to $25 \%$ have been reported. ${ }^{2-5}$ Consequently,

modifications of this procedure have been attempted to improve success rates. One study found that braided ultrahigh molecular weight polyethylene sutures provide superior mechanical characteristics to monofilament nylon in mechanical testing. ${ }^{6}$ Two subsequent reports found braided sutures to be mechanically superior to monofilament when testing toggle pin fixation for coxofemoral luxation ex vivo. ${ }^{7,8}$ Accordingly, numerous studies have described use of braided suture material systems for the treatment of coxofemoral luxation with success rates up to $94 \% .^{9-12}$

(c) 2018 Georg Thieme Verlag KG Stuttgart · New York
DOI https://doi.org/ 10.1055/s-0038-1667064. ISSN $0932-0814$.
October 10, 2017

accepted after revision

May 9, 2018 
In addition to using mechanically superior materials for toggle fixation of coxofemoral luxation, some surgeons have attempted to improve surgical technique. The currently recommended procedure involves drilling the femoral and acetabular bone tunnels separately. This typically involves retracting the femoral head caudal to the acetabulum to facilitate drilling a hole in the acetabular fossa. In cases of craniodorsal luxation, retraction of the femoral head caudal to the acetabulum can be challenging. Once the acetabular tunnel is drilled, the toggle is then passed through the hole in the acetabular fossa and seated on the medial side of the acetabular wall. The remaining suture is passed through the femoral bone tunnel from the femoral head onto the proximolateral femur using a suture passer. The joint is then reduced, the suture ends threaded through a button on the proximolateral femur and the suture knotted. ${ }^{13}$

In an effort to reduce surgical trauma and time, as well as potentially minimizing contamination of the implants, some surgeons have drilled the acetabular tunnel through the femoral tunnel. In fact, drilling of both the femur and acetabulum simultaneously while using a special aiming guide was used in the first report of toggle pin fixation. ${ }^{1}$ Similarly, one case report described acetabular drilling through the femoral bone tunnel under arthroscopic guidance. ${ }^{9}$ Yet another study developed a fluoroscopic-guided technique for drilling both the femur and acetabulum together. ${ }^{14}$ More recently, some surgeons have begun drilling the acetabulum through the femoral tunnel without a jig or arthroscopic or fluoroscopic guidance (J. Suber, DVM and C. Walls, DVM, personal communication, 2015). The femoral head is reduced after drilling the femoral bone tunnel and the limb is held slightly abducted with neutral flexion/extension. A long arthroscopic probe (Canine 2.6-mm Arthroscopic Switching Stick; Arthrex Vet Systems, Naples, Florida, United States) is used to palpate the articular surface of the acetabulum as well as the non-articulating portion of the fossa. When the femoral tunnel is lined up with the acetabular fossa, the drill bit is re-placed down the femoral tunnel and the acetabular fossa is drilled blindly. The limb is maintained in this position and the femoral and acetabular tunnels may be palpated again with the long arthroscopic probe to ensure tunnel alignment. The toggle and attached suture are passed from the proximolateral femur through the femoral and acetabular holes using a commercially available dedicated toggle delivery device (Button Inserter; Arthrex Vet Systems, Naples, Florida, United States). This technique eliminates the need to retract the femoral head caudal to the acetabulum and does not require a jig, fluoroscopy or arthroscopic assistance. In addition, it simplifies insertion of the suture to a single step, potentially decreasing surgical time, exposure needed and possible contamination of implants.

Although the simplified characteristics of this modified technique are appealing, several questions exist, including (a) does this drilling technique risk damaging the acetabular cartilage? (b) what is the success rate of deploying the toggle through the femur and acetabulum in a single manoeuvre? and (c) is this technique faster than the standard technique? The purposes of this cadaveric study were to evaluate the safety and efficacy of this modified technique compared with the standard technique using a braided suture and toggle system (Tightrope; Arthrex Vet Systems, Naples, Florida, United States) in which the toggle has a diameter of $\sim 2.9 \mathrm{~mm}$ at its widest dimension. We hypothesized that both techniques would result in holes within the acetabular fossa without cartilage damage. We also hypothesized that use of the novel delivery device would result in accurate placement of the toggle on the medial acetabular wall in all cases. Finally, we hypothesized that the modified technique would be faster than the standard technique.

\section{Materials and Methods}

\section{Cadaver Preparation}

Sixteen cadavers from skeletally mature dogs weighing greater than $20 \mathrm{~kg}$ and euthanatized for reasons unrelated to this study were collected. The cadavers were eviscerated and transversely sectioned in the proximal lumbar region to create a pelvic 'specimen' that included the pelvis and pelvic limbs. The pelvic specimens were then stored at $-10^{\circ} \mathrm{C}$ and thawed to room temperature just prior to use.

\section{Study Design}

Coxofemoral luxations were repaired bilaterally by toggle pin technique by a surgical resident ( $n=8$ cadavers) and a diplomate surgeon ( $n=8$ cadavers). For each specimen, a 'standard' technique was performed on one hip in which the femoral and acetabular tunnels were drilled independently and a 'modified' technique was performed on the contralateral side where the acetabular hole was drilled via the femoral tunnel. Technique order and side (right vs left) were decided with coin tosses. Dogs with evidence of coxofemoral osteoarthritis (femoral head flattening, cartilage wear, osteophytosis) identified during the procedure were excluded from the study.

\section{Surgical Technique}

For both the 'standard' and 'modified' procedures, a craniolateral approach to the hip joint with partial tenotomy of the deep gluteal tendon was performed. Transection of the round ligament and disarticulation of the femoral head was performed using a 22.8-cm Hatt spoon (Hatt spoon; Integra Miltex, Plainsboro, New Jersey, United States) with a $15-\mathrm{mm}$ oval cup. The femur was then externally rotated to luxate the hip. Shallow 2.0-mm starter holes were drilled in the fovea capitis and the proximo-lateral aspect of the femur to help maintain placement of a C-shaped aiming guide (Femoral Aiming Guide; Arthrex Vet Systems, Naples, Florida, United States) and a $1.25-\mathrm{mm}$ diameter Kirschner wire (Kirschner Wire; Arthrex Vet Systems, Naples, Florida, United States) was then driven from the proximo-lateral aspect of the femur through the femoral neck to exit the fovea capitis. Placement of the Kirschner wire was checked to make sure the exit point was located at the fovea capitis. If it were not, the Kirschner wire was removed, and the process repeated until it was appropriately positioned. The femoral tunnel was then drilled over the Kirschner wire using a $3.5-\mathrm{mm}$ cannulated drill bit from the proximo-lateral aspect of the femur to the fovea capitis (3.5-mm cannulated drill bit, Arthrex Vet Systems, Naples, Florida, United States). The location 
selected for the exit point of the tunnel on the lateral femur was relatively proximal, just below the greater trochanter, a location selected to try and keep the trajectory of the femoral tunnel aimed relatively ventral, and hence into the acetabular fossa rather than dorsal into the articular cartilage.

\section{Standard Technique}

After drilling the femoral tunnel, the femur was retracted in a caudal direction by applying traction just distal to the femoral head with an Army-Navy retractor. Remnants of the round ligament were removed using a rongeur. A 3.5- $\mathrm{mm}$ drill bit was used to drill a hole in the dorsal aspect of the acetabular fossa. For the resident's 'standard' technique group, the toggle with attached suture was loaded onto the delivery device ( $\mathbf{- F i g . 1}$ ). The tip of the delivery device was placed into an indentation at the end of the toggle. A button on the handle of the delivery device retracts the tip, deploying the toggle. The toggle was passed from lateral to medial through the femoral bone tunnel. The toggle was deployed, grasped adjacent to the femoral head, pulled through the femoral tunnel further to provide adequate working length and reloaded onto the delivery device before being passed through the acetabular hole while an assistant continued to retract the femur caudally. Once through the acetabular hole it was deployed, and the suture was pulled taut to seat the toggle against the medial acetabular wall. The hip was then reduced, and the two suture strands were tied over the provided titanium 4-hole button on the proximolateral femur.

For the surgeon's 'standard' technique, the femoral head was reduced after both the femoral and acetabular tunnels were drilled separately. A long arthroscopic probe was placed through the femoral tunnel from lateral to medial and the limb was manipulated until the long arthroscopic probe entered the acetabular drill hole, confirming alignment of the two tunnels. Once located, the delivery device was used to pass the toggle and suture through the femoral bone tunnel, across the joint, and through the acetabular bone tunnel before deploying the toggle. The suture was pulled taut to seat the toggle on the medial acetabular wall before tying the suture over the 4-hole button on the proximolateral femur. This toggle passing technique was used to assess the ability to successfully pass and seat the toggle in a single manoeuvre even when the femoral and acetabular tunnels were drilled separately.

\section{Modified Techniques}

For the 'modified' technique, the femoral head was reduced into the acetabulum immediately following completion of the femoral bone tunnel. A long arthroscopic probe was passed through the femoral tunnel and used to palpate the acetabulum and the limb was manipulated in an effort to palpate the acetabular fossa. Emphasis was placed on keeping the limb abducted and otherwise at a neutral standing angle with regard to coxofemoral extension. This was done to keep the drill bit oriented ventrally and into the fossa. Once this positioning was obtained, ideally having confidently palpated the acetabular fossa, the limb was held in that position. A 3.5-mm drill bit was inserted through the femoral bone tunnel and the acetabulum was drilled blindly. The drill bit was removed, and the delivery device was used to pass the toggle through the femoral bone tunnel, across the joint and through the acetabular tunnel. The toggle was deployed, and the suture was pulled taut to seat the toggle on the medial acetabular wall. The suture was tied over the 4-hole button on the proximolateral femur.

\section{Outcome Measures}

Three outcome measures were used to compare the different techniques: time to perform the procedure, successful deployment of the toggle through both the femoral and acetabular tunnels (graded as successful or not successful) and placement of the acetabular drill hole within the acetabular fossa (graded as successful or not successful). Procedure time was recorded from the time the hip was luxated until completion of the procedure. After completion of procedures on both hips, the cadaver was placed into dorsal recumbency and the pubis was removed. The soft tissues were elevated from the medial acetabular wall, which was examined to determine if the toggle had successfully passed all the way through the acetabular tunnel. Complete seating of the toggle against the medial acetabular wall was considered a success, while placement of the toggle anywhere else was considered a failure. The suture was then transected, and the hip was reluxated for inspection of the acetabulum. A drill hole entirely within the fossa without damage to the cartilage was deemed a success, while a hole not entirely within the fossa which did damage the cartilage was considered a failure.

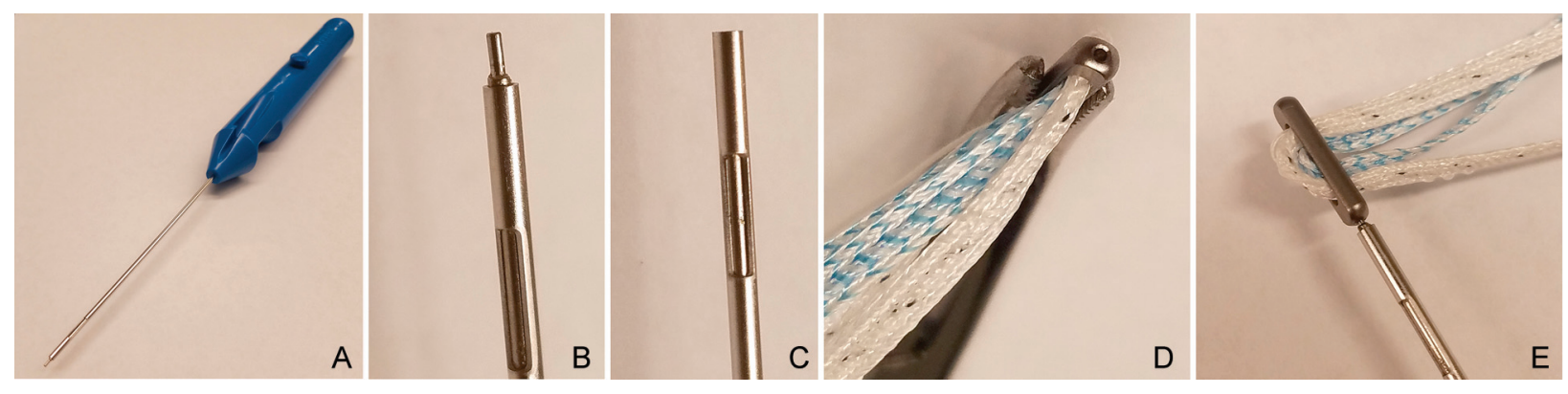

Fig. 1 (A) Toggle pin delivery device. (B) Tip of delivery device extended. (C) Tip of delivery device retracted. (D) Toggle being held with a haemostat showing indentation on one end. (E) Toggle and suture loaded onto delivery device. 


\section{Statistical Analysis}

Time for completion of the 'standard' and 'modified' procedures was compared for each surgeon using a paired $t$-test. Comparison of success and failure rates between the two techniques for acetabular drill hole placement and for seating of the toggle on the medial acetabular wall was evaluated using a Fisher's exact test.

\section{Results}

\section{Time to Completion}

None of the 16 cadavers were excluded from the study due to coxofemoral osteoarthritis. Time to complete the 'standard' technique was significantly longer than time to complete the 'modified technique' for both the resident (18.2 \pm 5.6 minutes vs. $13.8 \pm 2.8$ minutes; $p=0.04$ ) and the surgeon (mean: $19.4 \pm 5.5$ minutes vs. $12.9 \pm 2.7$ minutes; $p<0.01$ ).

\section{Drill Hole Location}

While palpating the articular cartilage through the femoral tunnel using the arthroscopic probe, in some specimens an obvious 'drop' was felt as the probe tip fell from the articular surface into the fossa. For other dogs, this 'drop' was less defined or non-palpable.

For the resident, separate drilling of the femur and acetabulum did not result in cartilage damage in any hips, while blind drilling of the acetabulum resulted in damage to two of eight hips. This difference was not significant $(p=0.466)$. There was also no cartilage damage when the femur and acetabulum were drilled separately by the surgeon, while cartilage damage occurred in four of eight hips with the modified drilling technique; this difference was not significant $(p=0.077)$. If cases performed by the resident and the surgeon were grouped, blind drilling of the acetabulum resulted in cartilage damage in 6 of 16 acetabula compared with 0 of 16 using the standard technique. This difference was significant ( $p=0.02$ ). Cartilage damage was located immediately caudal to the acetabular fossa in five cases ( - Fig. 2) and immediately dorsal to the acetabulum in one case.

\section{Seating of the Toggle}

For the resident, the toggle was firmly seated on the medial acetabular wall in seven of eight hips while passing the suture through the femur and acetabulum separately, and in eight of eight hips when passing the suture through the femur and acetabulum simultaneously; this difference was not significant $(p=1.0)$. In the single unsuccessful case, the toggle had not passed completely through the acetabular tunnel and upon tightening had become lodged in the tunnel with one end of the toggle protruding from the medial side of the acetabulum (-Fig. 3 ).

For the surgeon, who passed the suture through both tunnels simultaneously, the toggle was seated on the medial acetabular wall in 16/16 hips regardless of drilling method. If cases for the resident and surgeon were grouped and evaluated together, there was no significant difference in success rates between the toggle being passed in a 'one-step' or '2-step' process $(p=0.25)$.

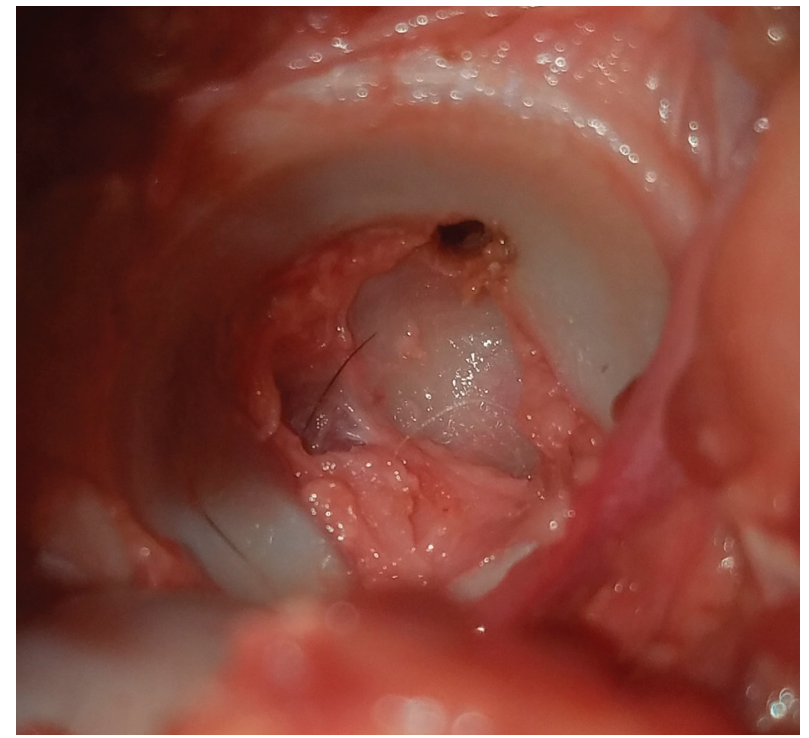

Fig. 2 A left acetabulum showing damage from drill bit penetration of the articular cartilage just caudal to the acetabular fossa after blind drilling through the femoral bone tunnel.

When passing the suture through the femur and acetabulum simultaneously, difficulty was encountered in locating the acetabular hole using the delivery device in two hips, one involving the resident and the other the surgeon. In these instances, the toggle could be passed through the femoral tunnel but was not lined up with the acetabular tunnel. The toggle was withdrawn while still attached to the delivery

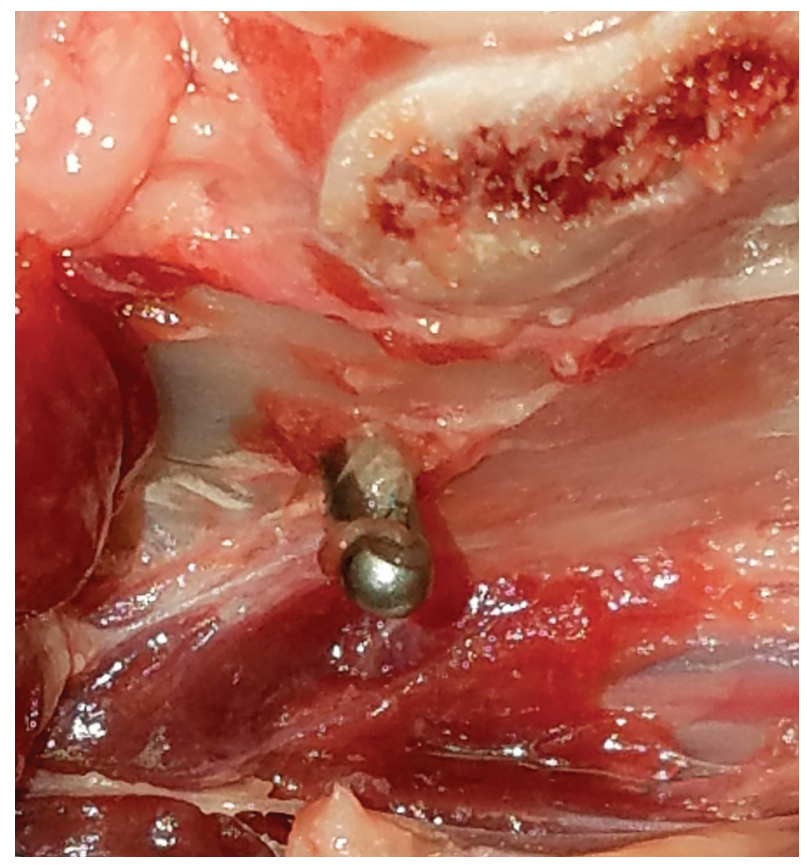

Fig. 3 Incomplete seating of the toggle on the medial acetabular wall. The toggle was not passed completely through the acetabular bone tunnel before deployment resulting in the toggle becoming lodged in the tunnel and one end protruding from the medial surface of the acetabulum. This occurred after drilling of the acetabulum independent of the femoral tunnel, and the suture was passed during direct visualization of the acetabular bone tunnel. 
device, and the arthroscopic probe was replaced through the femoral tunnel and limb manipulation performed until the femoral and acetabular tunnels were aligned. The toggle was then re-introduced using the delivery device. This occurred with one hip for the resident after drilling the femur and acetabulum together; this acetabular drill hole had not damaged the articular cartilage. On the third attempt, the toggle was eventually seated successfully on the medial acetabular wall. Placing the toggle all the way through the femur and acetabulum simultaneously was difficult in 1 of 16 hips for the surgeon where the acetabulum had been drilled independently of the femur. It took three attempts to successfully pass the toggle through the femur and acetabulum and successfully seat it on the medial wall.

\section{Discussion}

The results of this study revealed that although the modified drilling technique was faster than the standard drilling technique, it resulted in cartilage damage in 6 of 16 acetabula compared with no cartilage damage when drilling the femoral and acetabular tunnels separately. The incidence of cartilage injury between the two different drilling techniques was significant when results from the surgeon and resident were combined for analysis but was not significant when results from the surgeon and resident were analysed separately. The lack of a significant difference when the data were analysed separately is likely to be attributable to a type II statistical error and we believe the difference in cartilage injury is clinically relevant. Cartilage damage occurred with the blind drilling technique even though several steps were taken to reduce the chance of articular cartilage damage. One potential explanation for such failures was the inability to reliably palpate the acetabular fossa with the arthroscopic probe in some specimens. Failure to palpate a distinct drop of the probe into the fossa may be the result of a lack of defined fossa in some dogs or by the tissue that remains in the fossa when no approach to the acetabulum is performed to remove remnants of the round ligament. The effect of round ligament remnants in the fossa on drill hole location or femoral head reduction remains unclear. Another explanation for failure is that pelvic specimens of cadavers were used as opposed to whole cadavers in this study and such pelvic specimens were not firmly secured to the table. It is possible that when adjustments were made to the limb, such as external rotation and abduction to reorient the drill bit within the acetabulum, the low weight of the unsecured pelvic specimen allowed the pelvis to rotate with the limb, limiting the effectiveness of the positioning adjustments. Whatever the reasons, in our hands, blind drilling of the acetabulum through the femur is not adequately safe for use in clinical practice and independent drilling of each tunnel is recommended.

There are multiple methods that can be used to pass the toggle and associated suture through the femoral and acetabular tunnels. The most common method currently recommended involves passing the toggle through the acetabular hole, removing the button before withdrawing the suture material back through the femoral tunnel using a suture passer and then re-placing the button to secure the suture on the lateral aspect of the femur. ${ }^{13}$ As an alternative, passing the toggle through the lateral femur and acetabulum in a single manoeuvre is possible using the toggle delivery device. The toggle was successfully passed all the way through the femur and acetabulum in all 24 hips in which this technique was used, regardless of acetabular drilling technique. Hence, we conclude that this method for delivering the toggle is feasible and may have some clinical advantages over deployment of the toggle separately through the acetabular and femoral tunnels. Passing the toggle in a single manoeuvre may reduce surgical time and manipulation and thus the risk of contamination of the implant. However, these hypotheses would need to be tested since we did not compare this single manoeuvre technique with a technique in which the suture is pulled through the femoral tunnel using a suture passer. Furthermore, multiple attempts at passing the toggle, as occurred in two hips in our study, could add time to the surgical procedure rather than decrease time. It should also be noted that passing the toggle through the femoral and acetabular tunnels in a single manoeuvre requires that the femoral tunnel be of sufficient diameter to allow passage of the toggle. In some dogs, this size tunnel may predispose to femoral neck fracture and in such cases drilling a narrower femoral tunnel and passing the suture material through the femoral tunnel without the toggle may be desirable.

In conclusion, replacing the surgical step in which the acetabulum is exposed, the fossa cleared, and the acetabulum drilled under visualization with drilling of the acetabulum blindly through the femoral tunnel reduces surgical time. However, this is at the expense of an increased risk of damaging the acetabular cartilage and is not recommended. Passing of the toggle through the femur and acetabulum in a single manoeuvre is feasible using the commercially available toggle delivery device, regardless of whether the femoral and acetabular tunnels are drilled independently.

\section{Funding}

Materials used in this study were provided by Arthrex Vet Systems, Naples, Florida, United States.

\section{Conflict of Interest}

Dr. Franklin is a consultant for Arthrex Vet Systems.

\section{Author Contributions}

Samuel P. Franklin contributed to conception of study, study design, and acquisition of data and data analysis and interpretation. Jacob I. Helmick contributed to acquisition of data and data analysis and interpretation. Suzanne E. Bugbee and Jason R. Strasberg contributed to acquisition of data. All authors drafted, revised and approved the submitted manuscript.

\section{Acknowledgements}

The authors would like to thank Lynn Reece, Brent Norwood, Jessica Posey and McKenzie Towe for their assistance with this project. 


\section{References}

1 Knowles AT, Knowles JO, Knowles RP. An operation to preserve the continuity of the hip joint. J Am Vet Med Assoc 1953;123(921): 508-515

2 Beckham HP Jr, Smith MM, Kern DA. Use of a modified toggle pin for repair of coxofemoral luxation in dogs with multiple orthopedic injuries: 14 cases (1986-1994). J Am Vet Med Assoc 1996;208(01): 81-84

3 Demko JL, Sidaway BK, Thieman KM, Fox DB, Boyle CR, McLaughlin RM. Toggle rod stabilization for treatment of hip joint luxation in dogs: 62 cases (2000-2005). J Am Vet Med Assoc 2006;229(06): 984-989

4 Evers P, Johnston GR, Wallace LJ, Lipowitz AJ, King VL. Long-term results of treatment of traumatic coxofemoral joint dislocation in dogs: 64 cases (1973-1992). J Am Vet Med Assoc 1997;210(01): 59-64

5 Bone DL, Walker M, Cantwell HDAN. Traumatic coxofemoral luxation in dogs results of repair. Vet Surg 1984;13(04): 263-270

6 Rose ND, Goerke D, Evans RB, Conzemius MG. Mechanical testing of orthopedic suture material used for extra-articular stabilization of canine cruciate ligament-deficient stifles. Vet Surg 2012; 41(02):266-272

7 Zambrano S, Teixeira MW, Lamb JL, et al. Comparison of three toggle suture constructs (TSCS) for craniodorsal hip luxation in dogs: an ex-vivo biomechanical study. Abstracts presented at: 43rd Annual Conference of the Veterinary Orthopedic Society; February 27-March 5, 2016; Big Sky, Montana

8 Jha S, Kowaleski MP. Mechanical analysis of twelve toggle suture constructs for stabilization of coxofemoral luxations. Vet Surg 2012;41(08):948-953

9 Lee H, Heo S. Minimally invasive arthroscopic- assisted reduction with TightRope in a dog with coxofemoral luxation. Pak Vet J 2013;34(04):551-553

10 Tamburro R, Carli F, Cinti F, Puggioni A, Venturini A. Caudal approach and Mini TightRope system (mTR) application for the treatment of craniodorsal hip luxation in a cat: a case report. Vet Med (Praha) 2013;(09):500-504

11 Kieves NR, Lotsikas PJ, Schulz KS, Canapp SO. Hip toggle stabilization using the TightRope ${ }^{\circledR}$ system in 17 dogs: technique and longterm outcome. Vet Surg 2014;43(05):515-522

12 Ash K, Rosselli D, Danielski A, Farrell M, Hamilton M, Fitzpatrick N. Correction of craniodorsal coxofemoral luxation in cats and small breed dogs using a modified Knowles technique with the braided polyblend TightRope systems. Vet Comp Orthop Traumatol 2012; 25(01):54-60

13 Wardlaw JL, Mclaughlin RM. Toggle rod stabilization. In: Johnston SA, Tobias KM, eds. Veterinary Surgery Small Animal. Vol. 1. 2nd ed. St Louis, Missouri: Elsevier Inc.; 2018:962-963

14 Serdy MG, Schulz KS, Hornof W, Koehler C, Chiu D, Vasseur PB. Closed toggle pinning for canine traumatic coxofemoral luxation. Vet Comp Orthop Traumatol 1999;12(02):6-14 